\title{
Principles of visual motion detection
}

\author{
Alexander Borst and Martin Egelhaaf
}

Motion information is required for the solution of many complex tasks of the visual system such as depth perception by motion parallax and figure/ground discrimination by relative motion. However, motion information is not explicitly encoded at the level of the retinal input. Instead, it has to be computed from the time-dependent brightness patterns of the retinal image as sensed by the two-dimensional array of photoreceptors. Different models have been proposed which describe the neural computations underlying motion detection in various ways. To what extent do biological motion detectors approximate any of these models? As will be argued here, there is increasing evidence from the different disciplines studying biological motion vision, that, throughout the animal kingdom ranging from invertebrates to vertebrates including man, the mechanisms underlying motion detection can be attributed to only a few, essentially equivalent computational principles. Motion detection may, therefore, be one of the first examples in computational neurosciences where common principles can be found not only at the cellular level (e.g. dendritic integration, spike propagation, synaptic transmission) but also at the level of computations performed by small neural networks.

Thinking about how the nervous system extracts motion information, one might imagine that it compares successive images to measure the displacement of a certain object. This implies that the identification of specific features in a scene is a prerequisite for the perception of motion. Although feature identification may play a role in motion vision under certain circumstances ${ }^{1}$, it is not necessary. This could be shown in psychophysical and behavioural experiments on various species by the use of periodic or statistical patterns containing no prominent feature ${ }^{1,2}$. It is now widely accepted on the basis of such experiments that motion is initially evaluated in parallel by two-dimensional, retinotopically organized arrays of local motion detectors, which operate in the simplest case directly on the local light intensity (for review see Ref. 3).

\section{Models of motion detection}

From a theoretical point of view, local motion detection mechanisms have to satisfy certain minimum requirements in order to signal motion in a directionally selective way (see Box 1). In brief, a movement detector has to be asymmetrical and needs at least two inputs, which interact in a non-linear way $^{4-6}$. These requirements are met by a variety of similar motion detection models ${ }^{6-10}$. Some of them characterize the computations underlying motion detection in formal terms, others try to account for them in terms of cellular mechanisms. Irrespective of the actual level of description, the various biological motion detection schemes have been divided into two main categories, the so-called gradient- and correlation-type models ${ }^{8,11}$. While in the gradient schemes an estimate of local motion is obtained by relating the simultaneously measured spatial and temporal changes in local light intensity of the moving image $^{7,8,11}$, in the correlation schemes ${ }^{2,6,12}$ and their mathematical equivalents ${ }^{13}$ this is done by evaluating a kind of spatiotemporal cross-correlation of the appropriately filtered signals originating from two points in the retinal image. The gradient scheme originated from the study of computer vision and was applied only later to biological motion vision ${ }^{7,8}$. In contrast, the correlation-type of movement detector was deduced from behavioural experiments on motion vision in insects ${ }^{2,6}$. Subsequently, it was successfully used to explain motion detection in vertebrates including $\operatorname{man}^{12,14-18}$. The well-known 'BarlowLevick'-type of movement detector, which was originally proposed to account for motion detection in the rabbit retina ${ }^{19}$, belongs also to the broad class of correlation-type movement detectors in that it is a variant of them, being effected by logical operations rather than in an analogue form.

\section{Box 1. General Requirements for a Directionally Selective} Movement Detector ${ }^{4-6}$

\section{Two Inputs}

Two inputs are necessary since motion is a vector that needs two points for its representation. A single photoreceptor could not distinguish a dark bar that crossed its receptive field from the left to the right from one that crossed from the right to the left, or from a momentary dimming of the light.

\section{Non-linear Interaction}

A non-linear interaction between the input signals is required. Otherwise, the timeaveraged output of a detector would be equal to the detector response to its time-averaged input signals. In the averaged input signals, however, all information about the temporal sequence is lost. Thus, a movement detector with a linear interaction cannot be directionally selective.

\section{Asymmetry}

The two input signals of a movement detector have to be processed in a slightly different way. If the detector were symmetrical, its input channels could be interchanged without affecting its output. It is then no longer possible to discriminate which channel was excited first and which later. Accordingly, the detector would not be directionally selective.
Alexander Borst and Martin Egelhaaf are at the Max-PlanckInstitut für biologische Kybernetik Spemannstraße 38, D-7400 Tübingen, FRG. 
A
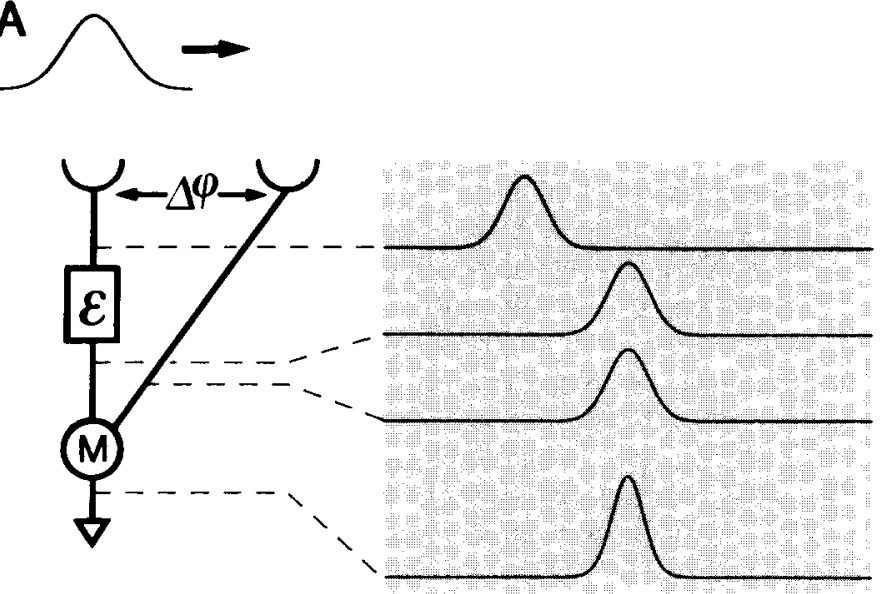

B

Null Direction

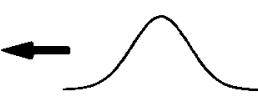

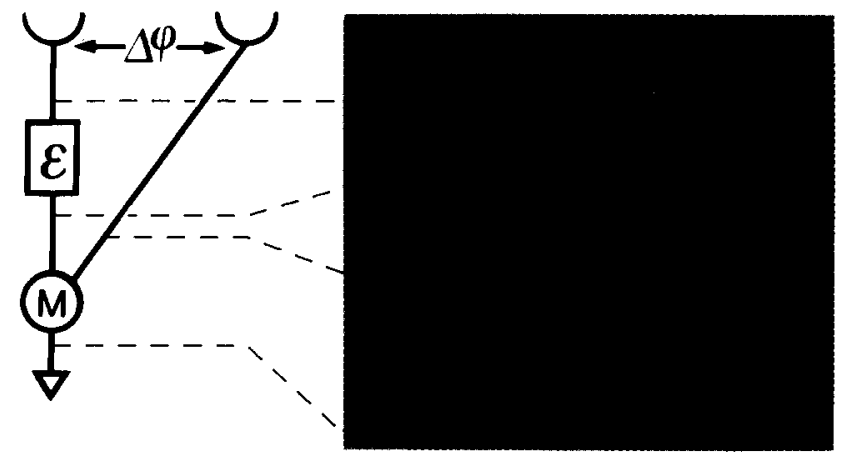

C
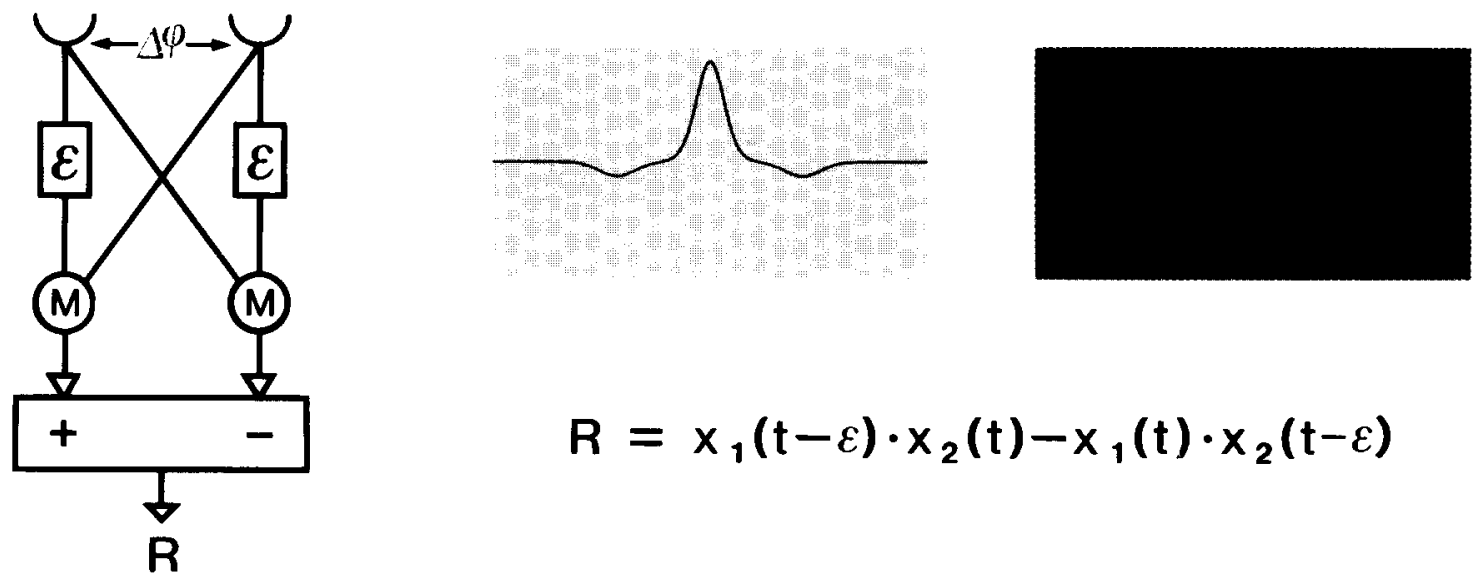

$R=x_{1}(t-\varepsilon) \cdot x_{2}(t)-x_{1}(t) \cdot x_{2}(t-\varepsilon)$

Fig. 1. Computation of motion information by a correlation-type movement detector. This detector consists of two mirror-symmetrical subunits (see C). In its simplest form, its input is given by the light intensities as measured at two points in space. In each subunit, the detector input signals are multiplied $(M)$ with each other after one of them has been delayed by a time interval, $\epsilon$. Both subunit outputs are then subtracted to give the final output signal of the detector. To facilitate an understanding of the different operations performed by a movement detector, the output of a single detector subunit to motion in opposite directions is considered first. The signals conveyed at the different stages of the detector subunit are indicated. When the stimulus pattern passes the two detector input channels, they are activated one after the other with a certain time shift. (A) When the pattern moves in the detector's 'preferred direction' (pale tint), the temporal separation of the signals in both input channels may be compensated for by the delay in the left arm of the detector. In this way both signals may coincide at the multiplication stage giving rise to a large output signal. (B) When the stimulus moves in the 'null direction' (dark tint), the temporal sequence of the signals in both channels is reversed. The delay increases their separation in time of arrival at the multiplication stage, which results in two small response peaks. (C) In a correlation-type movement detector two mirror symmetrical subunits are combined. By subtracting the output signals of both subunits, those response components are eliminated that are due to correlated input signals independent of the direction of motion and still present in the subunit output. When the subtraction stage is perfectly balanced, the responses to motion in opposite directions have the same amplitude and time course but different signs.

The gradient scheme, at least in its mathematically perfect form, obtains an exact measurement of the. local velocity $\mathrm{d} x / \mathrm{d} t$ by dividing the temporal gradient $\mathrm{d} I / \mathrm{d} t$ by the respective spatial gradient $\mathrm{d} I / \mathrm{d} x$ of the pattern ( $x$ and $t$ refer to the spatial variable and time, respectively; $I$ denotes the light intensity). If the spatial and temporal gradients are evaluated by finite mechanisms $^{5,7}$, the detector may acquire different properties. This, however, has not yet been investigated thoroughly.

The basic operations of a correlation-type movement detector are summarized in Fig. 1. In its simplest form, it operates directly on the retinal light intensity distribution or filtered versions of it (see below) and assumes a multiplication (which is the nonlinearity of the lowest possible order) for the interaction of its two input channels. These are spatially separated by the 'sampling base' $\Delta \varphi$ of the detector. When the signal in the input channel, which is activated first by a moving stimulus, is delayed by an appropriate time interval, $\epsilon$, the signals in both input channels tend to coincide at the multiplication stage $M$ resulting in a large response amplitude (Fig. 1A). Conversely, when the temporal sequence of stimulation is reversed (corresponding to motion in the opposite direction) the separation of both signals is further increased by detector delay, resulting in only smali responses (Fig. 1B). It is obvious that the 
delay and the sampling base determine the optimal velocity of the detector and, consequently, its dynamic range. The smaller the delay, the faster the optimal velocity and vice versa. The combination of a temporal delay and a multiplication of the detector input channels is the reason why this type of detector measures the degree of coincidence of the signals at its input stages or, in other words, performs a kind of spatiotemporal cross-correlation. However, a motion detector as shown in Fig. 1A, B also contains response components that are not specifically a result of the stimulus motion but are induced by correlated input signals that are independent of motion, such as background luminance. To eliminate these, a correlation-type movement detector is composed of two mirror-symmetrical subunits, each consisting of a separate delay unit and multiplication stage. The outputs of both subunits are then subtracted (Fig. 1C) leading to responses of the same amplitude but of different signs for motion in opposite directions. However, this

is only true if the movement detector is mathematically perfect, an unlikely assumption given the properties of the neuronal hardware. Thus a biological movement detector may not be strictly selective for motion, but can be expected to respond, at least to some extent, to temporal brightness modulations of a stationary stimulus ('flicker stimulation') ${ }^{20}$.

Various cellular models have been proposed which approximate, to some extent, the multiplication in the movement detector by specific cellular interactions ${ }^{9,10,21}$. However, since the same computation may be realized in different organisms by different cellular and synaptic mechanisms and, with a few exceptions, experimental data are missing, a comparison between different species is greatly facilitated by an analysis at the computational level. Therefore, we will base our predictions on the formal movement detector models and, in particular, on the correlationtype model.

\section{Experimental methods to study motion detection}

Motion detection has been studied by various stimulation methods. Very popular are dots or bars flashed sequentially at different positions and with different time intervals, thereby mimicking apparent motion. As a result of the non-linear interaction, maximum responses are obtained for certain spatial and temporal separations between the two stimuli, which then may be used to estimate the sampling base and the temporal delay of the underlying motion detection mechanism. This technique has been applied, for instance, to the rabbit retina ${ }^{19}$, to visual cortical areas of cats and monkeys ${ }^{22-24}$, and to the fly visual system ${ }^{25,26}$. Despite its appeal for many investigators, this approach may have some disadvantages. If one is interested in how the non-linear interaction of the movement detector input signals can be formally characterized, the responses obtained in this way are difficult to interpret analytically: because of their very transient nature, the time course of flashed stimuli will already be considerably altered by peripheral processing stages. As a consequence, the shape of the input signal to the essential non-linear interaction of the detector can hardly be inferred. This, of course, severely complicates an interpretation of the resulting output signals. Instead, when a grating pattern with a sinusoidally modulated brightness and moderate contrast is moved across the receptive field of a detector, the peripheral processing may, to a first approximation, behave linearly ${ }^{27-29}$. The actual input signals to the motion detector can then be assumed also to be sinusoidal ${ }^{20}$ and predictions can be derived for the response of a correlation-type movement detector (Box 2). These can be compared with experimental results obtained in studies using several different methods.

\section{Local motion detectors and consequences of spatial integration}

Individual movement detectors of the correlation type do not provide an exact estimate of the local pattern velocity. Their response to pattern motion, even of constant velocity, is not constant but modulated over time with the time course depending on both the velocity and the texture of the pattern ${ }^{20,30}$ (Box 2). Probably the simplest means of getting rid of these phase-dependent modulations is some sort of temporal or spatial integration over sufficiently large patches of movement detectors ${ }^{30}$. This is because the 
A

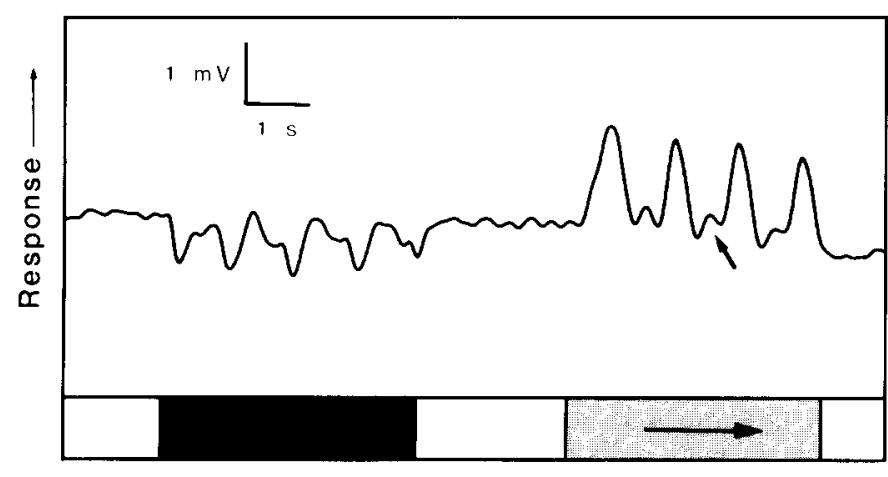

Time $\longrightarrow$

\section{Local Detector Response}

C Cat Simple Cell

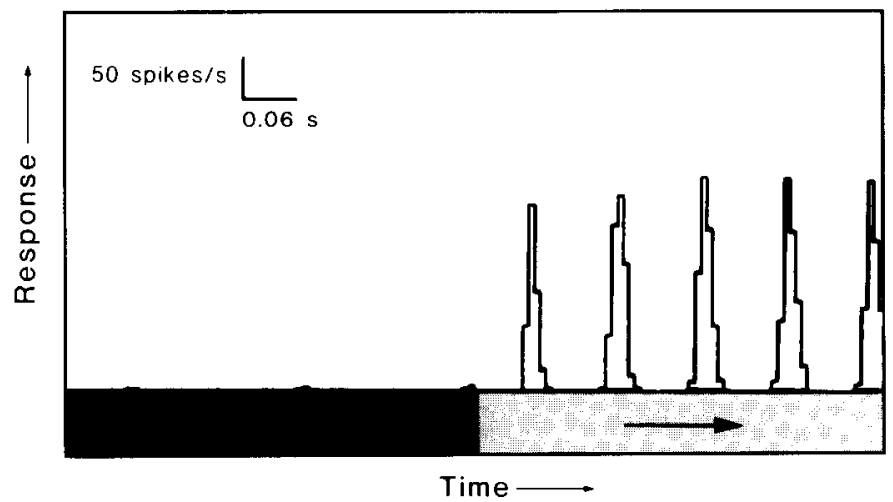

Integrated Detector Response

B

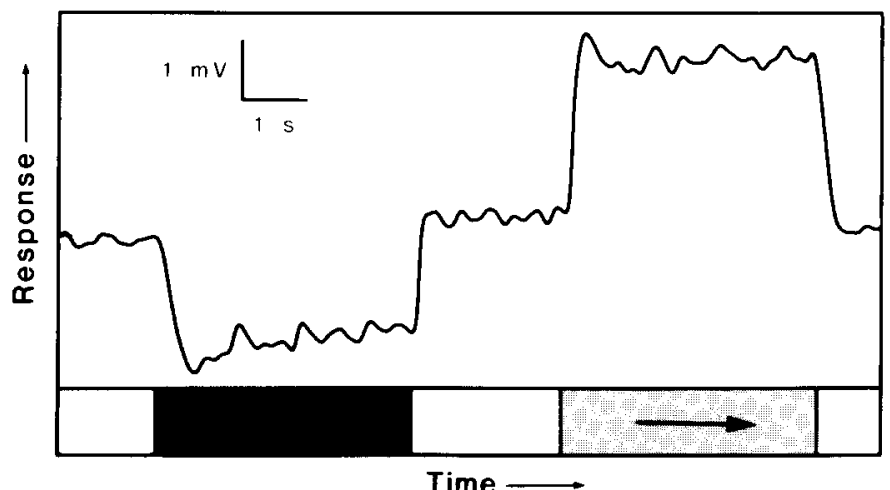

D

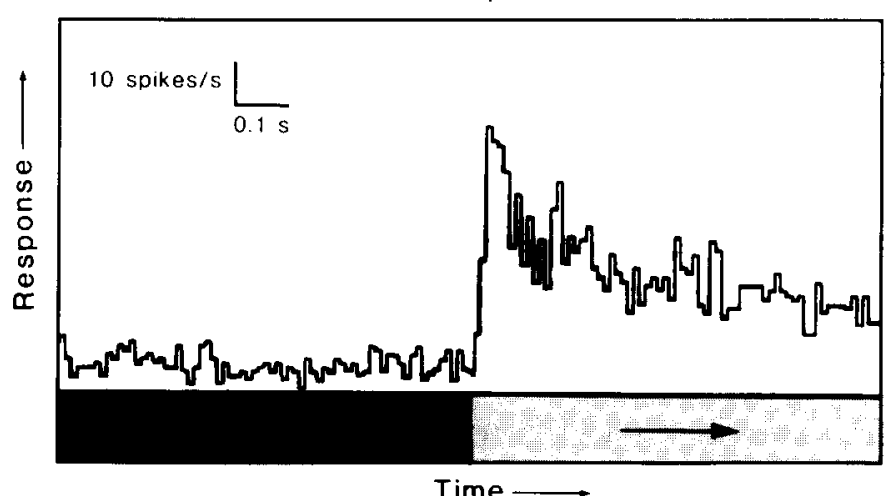

Fig. 2. Local and spatially integrated responses of biological motion detectors to sinusoidally modulated gratings drifting with a constant velocity. Graded changes of the membrane potential as recorded intracellularly from the direction-selective motion-sensitive HS-cell in the third visual ganglion of the fly (A, B) are compared with the visually induced spike activity of motion-sensitive cells in the cat visual cortex (C, D). (A) In the fly HS-cell, which integrates local movement detectors from large parts of the visual field, temporally modulated responses are obtained when spatial integration is prevented. This is achieved by moving the sine wave grating behind a small vertical slit, so that only a fraction of a spatial wavelength is seen by the fly at a given time 20 . Thus all vertically aligned local movement detectors are stimulated almost in synchrony. (B) When large parts of the receptive field of the cell are stimulated the response modulations disappear ${ }^{20}$. These findings are predicted on the basis of the movement detection model (Box 2). Note that the cell is, on average, depolarized by motion in its preferred direction (pale tint) and hyperpolarized by motion in the reverse direction (dark tint). (C) In the visual cortex of the cat, temporally modulated responses to a moving grating are found in simple cells, whereas unmodulated responses are common in complex cells (D). (Data shown in C and D from Ref. 38.) According to the predictions of the movement detector theory (Box 2), the modulated responses are interpreted as the output of local movement detectors, an interpretation that is consistent with the receptive field of simple cells consisting of separable on- and off-regions. In contrast, unmodulated responses are expected for the spatially integrated output of a detector array and, thus, correspondingly for complex cells with homogeneous on/off receptive fields integrating over elements with separable on/off regions. In the case of a pure multiplication such as movement detector non-linearity the response modulations of the local movement detectors should be composed of the fundamental and the second harmonic frequency of the temporal frequency of the stimulus (Box 2). In the fly, this prediction is satisfied ${ }^{20}$. In (A) the second harmonic component is indicated by an arrow. In the cat data (C) only the fundamental frequency component is visible. Note, however, the non-linearity introduced by the spiking threshold of the cell.

output signals of an array of movement detectors are all phase-shifted with respect to each other. Interestingly, spatial integration is quite common in cells that mediate information on motion both in vertebrates (e.g. Refs 31, 32) and in invertebrates (e.g. Ref. 33). In man, the importance of spatial integration for the perception of motion has been demonstrated in a number of psychophysical studies (e.g. Refs 34-36). It may suffice here to illustrate with two examples the responses of individual movement detectors and the consequences of spatial integration (Fig. 2).

In the cat visual cortex, the responses of directionally selective motion-sensitive simple cells (i.e. cells with separable on- and off-regions ${ }^{29,37}$ ) to the motion of periodic patterns are modulated in time (e.g. Ref. 38) (Fig. 2C). In contrast, complex cells that are known to combine output signals of several cells with antagonistic subfields ${ }^{22,37}$ exhibit unmodulated responses ${ }^{38}$ (Fig. 2D). This concurs with the predictions for the local and spatially integrated movement detector responses (Box 2).

In the insect visual system, we can, fortunately, investigate the responses of individual movement detectors and the consequences of spatial integration within one and the same neurone. The so-called HS-cells can be recorded intracellularly and identified individually in each animal by functional and anatomical criteria $^{39}$. Since these cells pool the signals of large retinotopic arrays of local movement detectors, one can obtain spatially integrated motion detector 
responses fairly easily. On the other hand, local detector responses are also accessible if spatial integration is prevented by presenting the stimulus pattern to the animal via a small slit ${ }^{20,30}$. The results of such an experiment are shown in Fig. 2A. They demonstrate that, as predicted by the correlation model and in agreement with data from motionsensitive cortical cells, (1) the responses of individual motion detectors are modulated in time, and (2) these temporal modulations disappear as a result of spatial integration (Fig. 2B). It should be noted that temporal modulations of local detector responses are not predicted by the gradient scheme, at least in its pure mathematical form.

We can now go one step further and characterize the non-linear interaction underlying motion detection on the basis of the time course of responses of individual movement detectors (Box 2). If the basic non-linearity in the motion detection system is of the second order, i.e. a multiplication, and no significant non-linear processing of the visual input takes place prior to or after motion detection, the response to a sine-wave grating moving with a constant velocity should contain only the fundamental and the second harmonic of the temporal frequency of the stimulus $^{10,20}$. This is indeed the case in the HS-cell of the fly which was analysed in this respect (Fig. 2A) ${ }^{20}$. The finding that the essential non-linearity responsible for movement detection in the fly is a multiplication is supported by a white noise analysis on another fly visual interneurone $\mathrm{e}^{40}$. Moreover, the same conclusion can be drawn for directionally selective motionsensitive cells in the visual cortex of the cat ${ }^{41}$. All these experiments strongly support a multiplicative interaction as the essential non-linearity underlying biological movement detection. Motion detection in quite different species may thus be based on essentially equivalent neuronal computations.

\section{Spatial filter properties and the problem of spatial aliasing}

A motion detector needs two input channels which are separated in space (see Box 1). The distance between them is often referred to as the "sampling base' of the detector. The sampling base, $\Delta \varphi$, determines the spatial resolution of the motion detection system and its dependence on the spatial frequency components of the stimulus pattern. The smallest spatial wavelength that is resolved adequately amounts to twice the sampling base, according to Shannon's sampling theorem ${ }^{42}$. For smaller wavelengths the response may become inverted, signalling the wrong direction of motion. This phenomenon is known as spatial aliasing ${ }^{43,44}$. Maximum responses are expected for wavelengths of four times the sampling base. Towards higher spatial wavelengths the response amplitudes decrease again. This means that, owing to its finite sampling base, a motion detection system has an intrinsic apparent spatial band-pass characteristic, even if there are no additional spatial filters in its input channels (Fig. 3). This fact is stressed here because it is often neglected when the spatial frequency dependence of motion detection systems is interpreted (e.g. Ref. 45).

To prevent spatial aliasing, high spatial frequencies should be removed from the detector input signals. This can be most easily achieved by additional spatial

\section{Receptive Field}

A

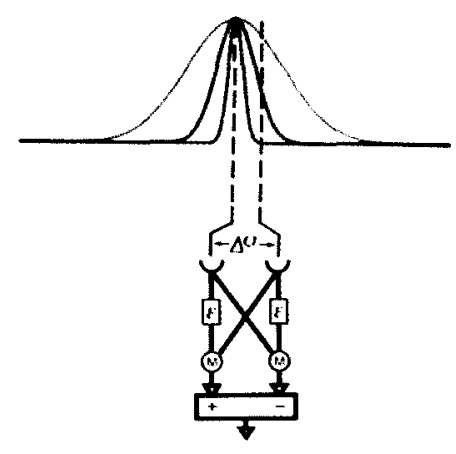

D

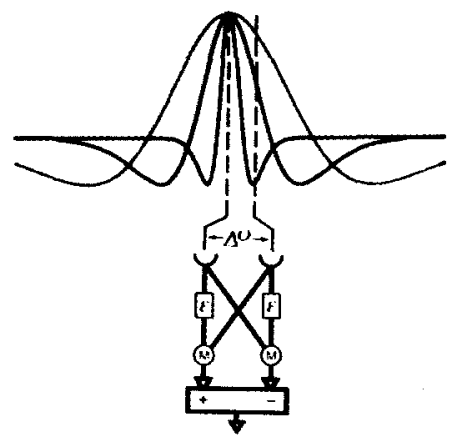

Contrast Transfer

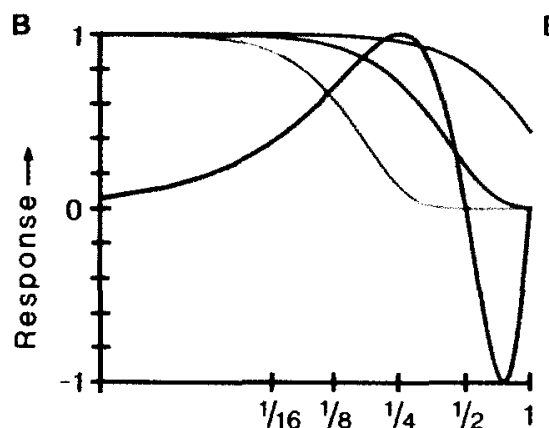

Spatial frequency $[1 \% / 1] \rightarrow$
Spatial trequency $[1 \% / 1] \rightarrow$

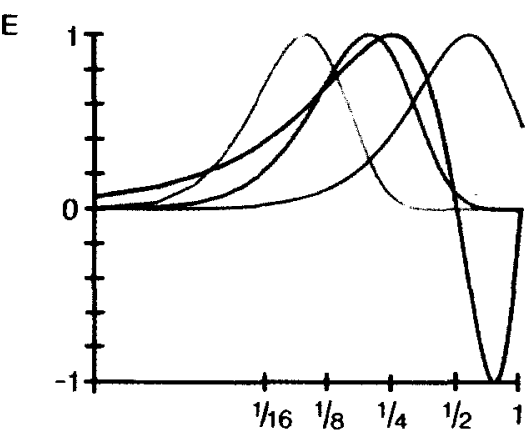

\section{Detector Response}
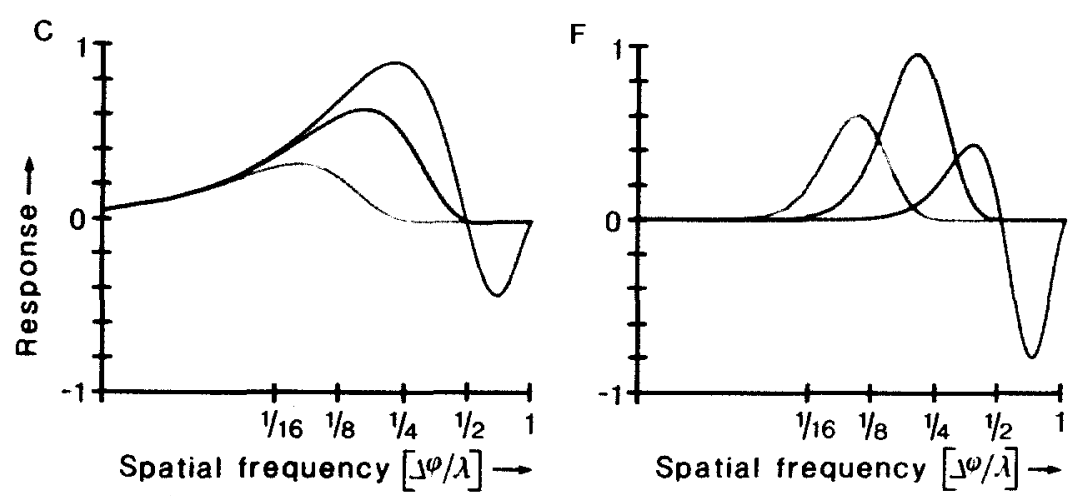

Fig. 3. Consequences of spatial filters in the movement detector input channels for the spatial frequency dependence of correlation-type movement detectors. (A-C) Consequences of Gaussian-shaped spatial low-pass filters with three different halfwidths (coloured lines) (modified from Ref. 44). (A) Receptive fields of the spatial filters in relation to the sampling base $(\Delta \varphi)$ of a movement detector. (B) Contrast transfer functions of the filters (coloured lines) together with the spatial frequency dependence of a movement detector without any input filter (shaded area). Note that for high frequencies the response is reversed due to undersampling ('spatial aliasing'). When the spatial frequency functions of both the filter and the motion detector are combined, responses as shown in (C) are obtained. The filter shown in blue is too narrow and, thus, does not prevent spatial aliasing, whereas the filter drawn in green is too broad, cutting out most of the detector's intrinsic optimal response range. The filter drawn in red is optimally matched to the sampling base of the detector. Experimental data on the acceptance angle of fly photoreceptors and the sampling base of its movement detection system show that in the fly both parameters are matched, as is expected for optimal imaging ${ }^{44}$. (D-F) Consequences of using spatial band-pass filters in the input lines of the motion detector instead of spatial low-pass filters as in (A-C). Again, the filter drawn in red is optimally matched, whereas the two others have a frequency optimum that is too low or too high for the sampling base and thus also for the intrinsic frequency dependence of the detector. Interestingly the optimal relation of $\Delta \varphi / \lambda$ is a quarter of the optimal spatial wavelength of the filter, which is approximately what has been measured in movement-sensitive neurons in the cat visual cortex ${ }^{23}$. 

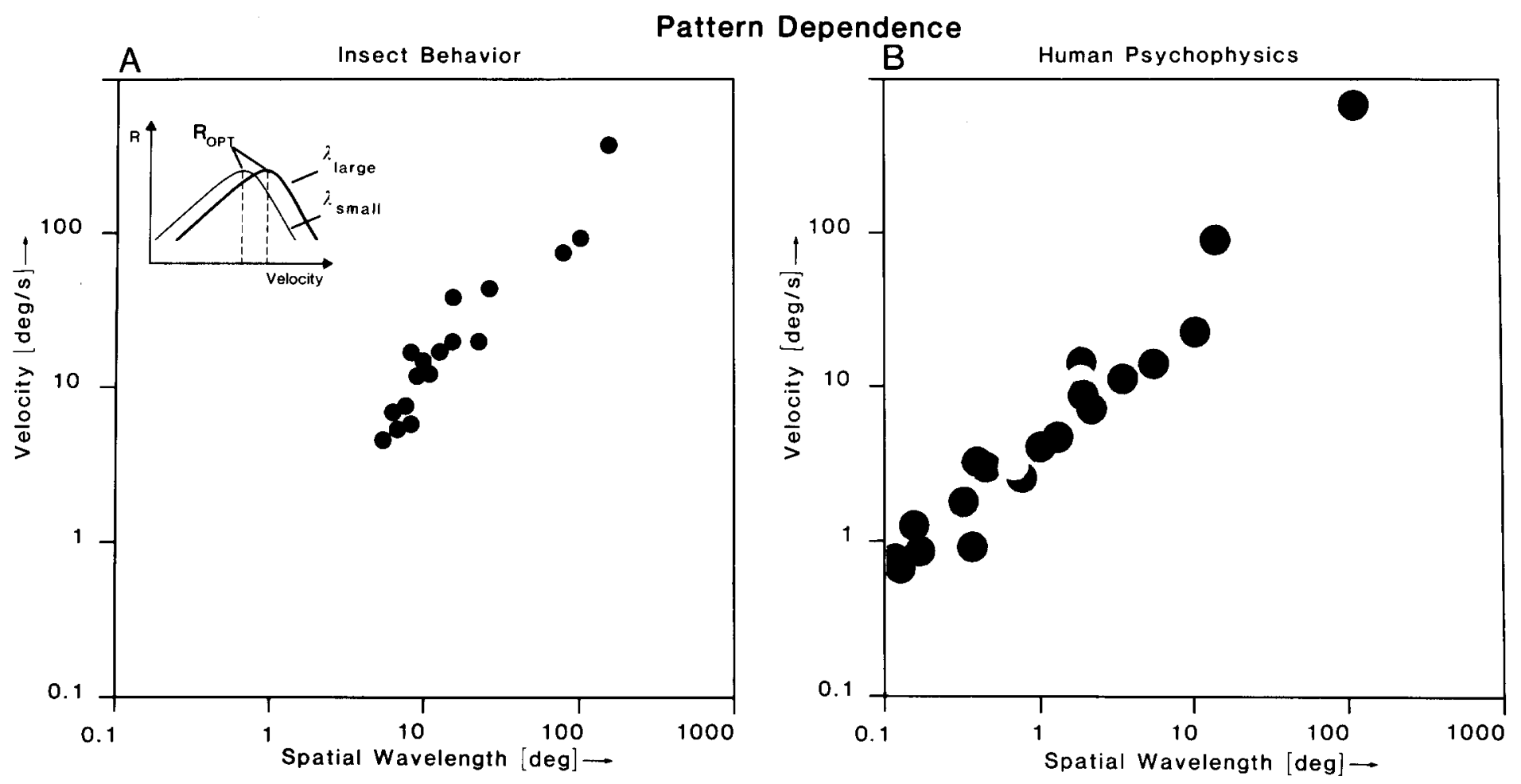

Fig. 4. Pattern dependence of different biological movement detection systems as revealed in optomotor turning responses in (A) the fly and (B) human psychophysics. To test whether or not movement detector responses are pattern dependent (see predictions in Box 2), mean response amplitudes to sine gratings of various spatial wavelengths drifting at different angular velocities were determined. For each wavelength they increase with increasing velocity, reach an optimum value and then decrease again. The response optima are shifted towards larger velocities for patterns with larger spatial wavelengths [shown schematically in the inset of (A)]. When the optimal velocity is plotted against the pattern wavelength the data points approximate to a straight line for motion perception of both insects and man. In correspondence with the model predictions, this indicates that the response optima are determined by the temporal frequency rather than by the velocity of the stimulus. Insect data represent the optomotor turning response of the fly ${ }^{44}$. Psychophysical data are compiled from various studies that use different indicators of movement perception: red ${ }^{54}$, yellow ${ }^{57}$ and violet circles ${ }^{55}$, maximum contrast sensitivity for the detection of a moving grating; blue circles ${ }^{58}$, maximum contrast sensitivity for estimating the correct direction of motion; orange circles $^{56}$, strength of motion after-effect as estimated by subjective scaling; green circles ${ }^{59}$, strength of motion after-effect as estimated by the velocity required to cancel it.

filters in the detector input lines. However, a detector elaborated in this way only shows an optimal performance if these spatial filters are matched appropriately to the detector's intrinsic spatial frequency characteristic. This means that, while filtering out those spatial frequencies that lead to aliasing, these filters should not attenuate the frequencies in the optimum operating range of the movement detector. This appears to be accomplished in insects and vertebrates in different ways.

In insects the sampling base is essentially determined by the spacing of the two-dimensional array of photoreceptors. Here, aliasing effects are dramatically alleviated simply by the bell-shaped sensitivity distributions of the photoreceptors which are a direct consequence of the optical properties of the eye (Fig. $3 A$ ). The width of the sensitivity distribution should be carefully matched to the sampling base ${ }^{44}$. If it is too small, then wavelengths that cause spatial aliasing may enter the motion detection system. On the other hand, if the sensitivity distribution is too broad, the motion detection system unnecessarily loses spatial acuity (Fig. 3B, C). A thorough analysis of the visual system of the fly revealed that the sampling base and the spatial low-pass filter properties of the receptors are almost optimally matched to guarantee a high performance in motion detection ${ }^{44}$.

In higher mammals including man there is good evidence for motion detectors with different sized sampling bases (e.g. Ref. 46). The sampling bases may be considerably larger than the angular distance between the photoreceptors and thus larger than the maximum resolution of the eye. Consequently, spatial aliasing cannot be prevented simply by using the spatial transfer properties of the optical apparatus. Instead, spatial frequency filters have to be generated by appropriate neuronal interactions. There is ample evidence that the visual system of different mammalian species acquires band-pass filter characteristics prior to the site of motion detection. These are generated by the antagonistic subfields found in the receptive fields of, for instance, retinal ganglion cells and cortical simple cells (Fig. 3D) ${ }^{29,37,47}$. Again, as in insects, the spatial filter properties of the input channels and the sampling bases of the subsequent motion detection system seem to be almost optimally matched (Fig. 3E, F). This can be inferred from data on directional-selective motion-sensitive simple and complex cells in the cat striate cortex ${ }^{23}$. There is a strong correlation between the spatial wavelength optimum of the cells and the optimum distance between two sequentially flashed bars simulating apparent motion. This optimum distance was found to increase proportionally with the optimum wavelength of the cell, yielding a mean ratio of about $1: 5$. A ratio of $1: 4$ is predicted as optimal on the basis of 
movement detector theory, if the optimum distance as determined in the experiments is assumed to correspond to the sampling base of the movement detector. Interestingly, corresponding results were found in related psychophysical experiments on motion vision in $\operatorname{man}^{48,49}$. In this way, the spatial frequency filters in the input channels to the movement detectors do not much attenuate frequencies in the detector's optimal operating range, but do prevent spatial aliasing. Since also low spatial frequencies are attenuated by the band-pass filter characteristics, the above data on motion-sensitive cells in the cat ${ }^{23}$ further indicate that motion detection is performed separately in different spatial frequency channels all tuned to the corresponding sampling bases. There is independent evidence from psychophysical experiments that this is also the case in man $^{50,51}$, and thus may reflect a general feature of motion detection at least in higher mammals. However, it should be emphasized that these differences between the motion detection system of insects and vertebrates only reflect their spatial input organization rather than any principal differences in the detection mechanism.

\section{Pattern dependence of motion detectors}

In contrast to the gradient scheme, which represents a pure velocity sensor, a motion detection mechanism of the correlation type does not correctly signal the local motion in the retinal image in terms of its direction and velocity. Instead, its output also depends in a specific way on the structure of the stimulus pattern, such as the spatial frequency content and contrast ${ }^{2,6,52}$. This characteristic can be used to distinguish this motion detection scheme from alternatives experimentally (Box 2) and may be illustrated most conveniently for grating patterns moving with a constant velocity. For a given spatial wavelength of the pattern, the response is expected to increase initially with increasing velocity until it reaches its optimum, and then to decrease again (see inset in Fig. 4). For a larger wavelength the response optimum should be shifted towards higher velocities in such a way that the ratio of the optimum velocity and the spatial wavelength of the pattern, i.e. the temporal frequency of the stimulus, is constant.

This prediction was originally tested in various insect species using a behavioural paradigm to monitor the output of their motion detection systems $^{52,53}$. Striped drums were rotated around the insect, while the compensatory optomotor turning responses were measured. Response optima for various combinations of pattern velocities and spatial wavelengths are drawn into a wavelength/velocity diagram (Fig. 4A). To a good approximation, they are located on a straight line. As predicted by model calculations, the response optima are thus essentially determined by the temporal frequency of the stimulus rather than by the pattern velocity itself.

Similar results have been obtained for humans in psychophysical studies using different indicators of the performance of the movement detection system $^{54-59}$, as well as in behavioural experiments on macaque monkeys ${ }^{60}$. The psychophysical results are summarized in Fig. 4B. As in insects, the response optima are located approximately on a common straight line in a wavelength/velocity diagram irrespective of the criteria used for the manifestation of human motion detection. This finding demonstrates that the motion vision systems of higher mammals including man are obviously incapable of yielding unambiguous information on velocity when exposed to grating patterns. Surprisingly, these results have not yet been discussed with respect to the mechanism underlying the evaluation of motion information. By analogy with the insect data, however, they provide strong evidence that in vertebrates too the initial representation of motion information is computed by a correlation-type movement detector. These findings corroborate those psychophysical and behavioural results that were interpreted explicitly in favour of essentially equivalent movement detection schemes in humans and insects $12,14,15,17,18$. It should be emphasized again, that this conclusion is not affected by the evidence for spatial band-pass filters in the movement detector input channels of man and higher mammals ${ }^{50,51}$. This only means that motion detectors with otherwise the same properties operate independently in different spatial frequency bands.

\section{Dynamical properties of motion detectors}

All the predictions discussed so far are concerned with the response of the motion detection system under steady-state conditions, i.e. when all stimulus parameters, such as pattern velocity are constant. The motion detection scheme discussed here to underly motion vision in different biological systems has limitations for the evaluation and representation of transiently changing motion stimuli ${ }^{61,62}$. These limitations are due to the fact that the signals in the two branches of each movement detector have to be phase-shifted with respect to each other for the movement detector to operate properly (Fig. 1). Analysing the transient response properties of movement detectors may, therefore, be interesting in two respects. First, they allow the assessment of how motion transients are represented at the output of a biological motion detection system. Second, they provide us with additional analytical tools to decide whether or not the motion detection system under investigation is of the correlation type.

In the following we focus on two types of transient stimuli, (1) an abrupt onset of motion of a sinusoidally modulated grating pattern, and (2) sinusoidal oscillations of this pattern with different frequencies. In Fig. 5, computer simulations of the spatially integrated output of an array of motion detectors are compared with the corresponding electrophysiological data obtained from wide-field movement-sensitive cells in the visual system of the fly. At the onset of motion, both the model and cellular responses reach their steady-state level only after some time. During the transition period the response oscillates with the temporal frequency of the stimulus (Fig. 5A). The oscillation amplitude decays exponentially with a time constant, which is determined by the movement detector delay ${ }^{62}$. When the pattern velocity changes sinusoidally (Fig. 5B), the time course of the response is smooth and follows the pattern motion quite well, as long as the oscillation frequency and amplitude are sufficiently small. For higher frequencies and amplitudes characteristic distortions in the response pro- 

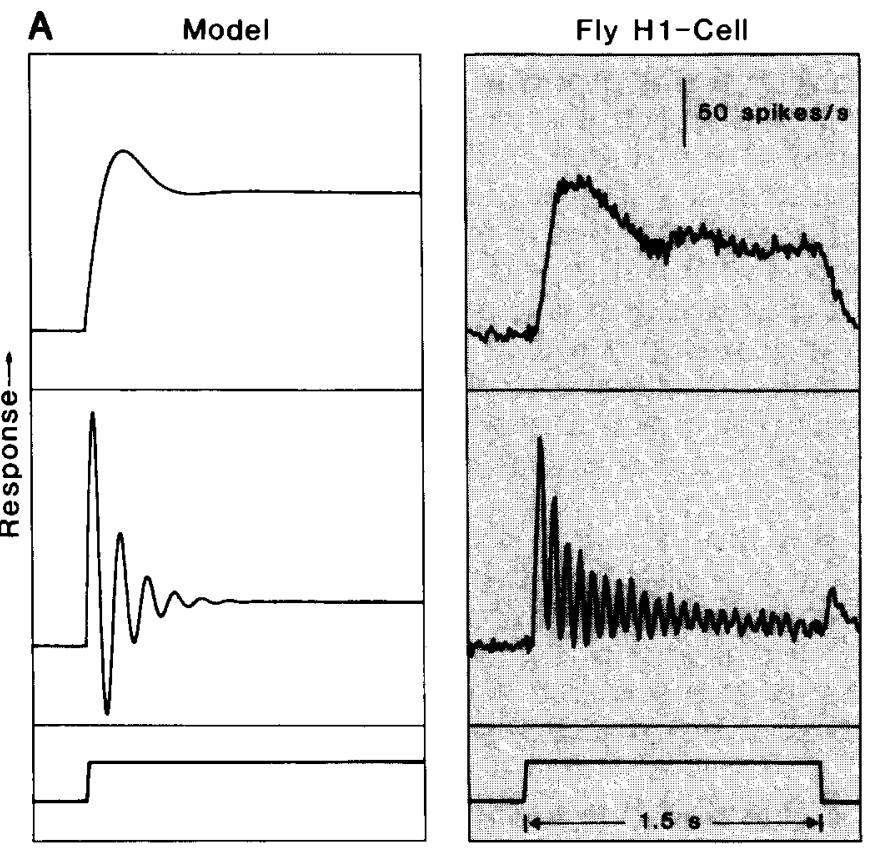

Time $\rightarrow$

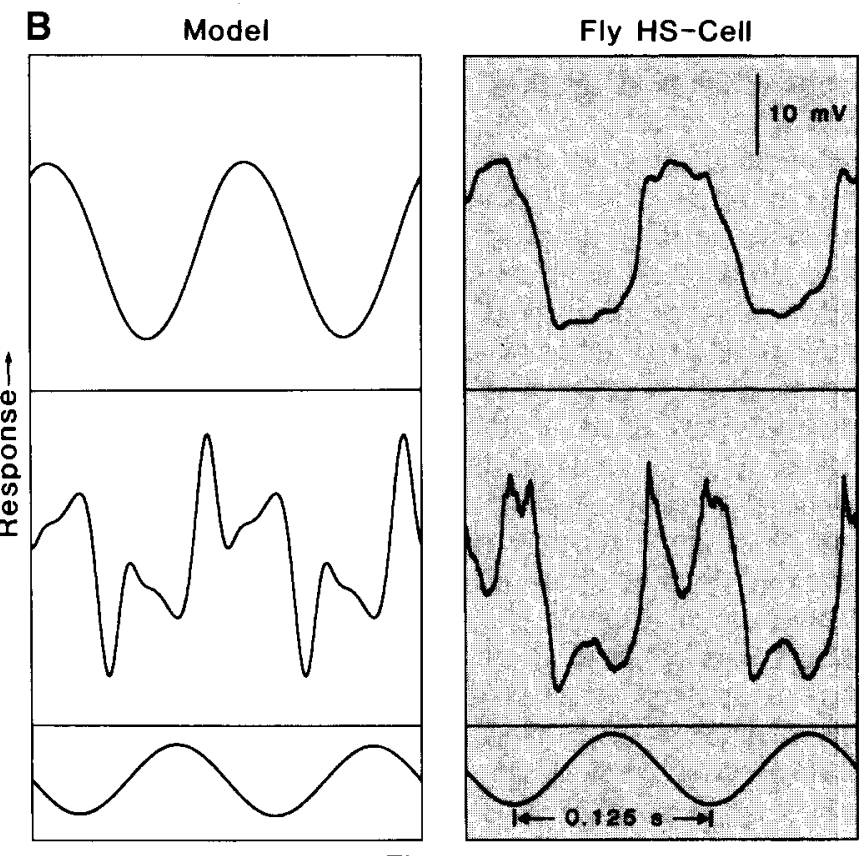

Time $\rightarrow$

Fig. 5. Transient movement detector responses. The diagrams on the left of both (A) and (B) are the simulated outputs of a spatially integrated array of correlation-type movement detectors. The diagrams on the right (tinted) are the corresponding responses of directionally selective large-field interneurons in the third visual ganglion of the fly. (A) Responses to the onset of motion of a grating with low frequencies (upper panels) and higher frequencies (middle panels). The onset and direction of the stimulus are shown in the lower panels. In the computer simulations (left) the grating frequencies were $2 \mathrm{~Hz}$ (upper panel) and $10 \mathrm{~Hz}$ (middle panel); the movement detector delay was approximated by a first-order low-pass filter with a time constant of $50 \mathrm{~ms}$. In the responses from the fly's $\mathrm{H} 1$-cell the grating frequencies were $2 \mathrm{~Hz}$ (upper panel) and $16 \mathrm{~Hz}$ (middle panel). Initially, the responses oscillate with the temporal frequency of the stimulus, until they settle to their steady-state level. This is due to the delay inherent in the detector. The oscillations are more pronounced for the higher frequency oscillations. (B) Graded membrane potential changes of an HS-cell in response to a sinusoidal stimulus grating oscillating with a frequency of $8 \mathrm{~Hz}$, but with different amplitudes of stimulus. In the upper panels of both the computer model and the fly's HS-cell, the amplitude of the stimulus grating was low $\left( \pm 2.5^{\circ}\right)$. In both, the responses follow the velocity modulations of the stimulus pattern (bottom panels) more or less smoothly. On the other hand, at higher oscillation amplitudes $\left( \pm 10^{\circ}\right.$, middle panels), characteristic distortions occur. In the computer simulations the time constant of the movement detector was $160 \mathrm{~ms}$; the oscillation amplitudes corresponded to the spatial wavelength (middle panel) and to one-fitth of the spatial wavelength (upper panel). In both the model and the experiment, the detector output signal is proportional to stimulus pattern velocity only within a limited dynamic range. (Modified from Refs $61,62$. )

files become visible in both the model and the cellular responses ${ }^{61}$. It can thus be concluded that the time course of the spatially integrated responses of correlation-type movement detectors and of the fly's movement detection system are proportional to pattern velocity only within a limited dynamic range of pattern motion, i.e. when the changes in pattern velocity are sufficiently small. Beyond this dynamic range, considerable deviations of the responses from the time course of pattern velocity occur.

Whether these conclusions also apply to motion detection in vertebrates cannot yet be decided, because comparable experimental data are not available in the literature. The predictions of characteristic response transients, as derived on the basis of the correlation-type of movement detector, represent another challenge to the conclusion that movement detection in insects and in the human visual system is based on essentially the same principles. In any case, the results shown in Fig. 5 suggest that the dynamics of detector responses should be considered if transient stimuli are used. In general, such data cannot be explained purely on the basis of the steadystate detector theory (compare e.g. Ref. 63 with Ref. 64). This might also be important when we interpret psychophysical and behavioural data because transient movement stimulation (such as brief stimulus presentation times occurring, for example, in reaction time measurements) is commonly used there and often leads to surprising results.

\section{Concluding remarks}

We hope to have illustrated that a clear understanding of the mechanisms underlying motion detection can only be achieved by a combined experimental and theoretical approach. It is important to know what information about the visual surround is extracted and represented by the mechanism if one wants to analyse the steps involved in processing this initial representation of local motion and which may give rise to the solution of more complex motion-dependent tasks.

To our knowledge, most relevant experimental data on biological motion detection now available are in accordance with the predictions of a correlation- 
type movement detector (Box 2). However, there exist some studies on human psychophysics ${ }^{65-67}$ that are interpreted as evidence in favour of the gradient scheme (e.g. see Ref. 8). However, the model predictions tested in these studies were not derived from the mathematically perfect gradient scheme but from a discrete version of it which was first proposed by Marr and Ullman ${ }^{7}$. There are two points to note here. (1) This discrete version of the gradient scheme no longer represents a pure velocity sensor, but instead, its response depends on the structure of the pattern, as is also characteristic of the correlation model (see Box 2). (2) The non-linear interaction inherent in this model relies on a logic AND-gate or, if implemented in an analogue way, on a multiplication ${ }^{7}$. Since this kind of interaction forms the core of the correlation-type of movement detector, the antagonism between the two formal rival models begins to fade when more realistic implementations of the gradient scheme are taken into account. Although many questions still remain, this is a further indication in favour of basically the same motion detection scheme occurring in insects and in different vertebrate species including man. Of course, this notion does not exclude species differences in the peripheral pre-processing of the signals on which the motion detection mechanism operates (see above).

Having characterized the basic computations underlying biological motion detection and, in particular, the non-linear interaction of the movement detector input channels, it might be of great interest for neurobiologists to account for them in cellular terms. For instance, different types of specific synaptic interaction, both inhibitory and excitatory, have been proposed theoretically as cellular mechanisms underlying directional selectivity. Interestingly, both types of interaction may approximate a multiplication which represents the core of a correlation-type movement detector ${ }^{9,10,21,68}$. Moreover, promising attempts have been made to unravel these synaptic interactions by experimental means in suitable systems such as the amphibian and rabbit retina ${ }^{69,70}$, the visual cortex of cats $^{71}$ and the fly visual system ${ }^{72,73}$. So there is hope that, in the near future, we will see how the different neuronal computations postulated by the correlation model are accomplished by the nervous system in terms of synaptic interactions.

Whatever the cellular mechanisms underlying movement detection in different species might be, the conclusions drawn above suggest that common principles of information processing exist not only at the level of properties of single nerve cells but also at the level of computations performed by neural circuits. Up to now there have not been many examples where this could be shown.

\section{Selected references}

1 Braddick, O. J. (1980) Philos. Trans. R. Soc. London Ser. B. 290, 137-151

2 Reichardt, W. (1961) Sensory Communication (Rosenblith, W. A., ed.), pp. 303-317, MIT Press and John Wiley $\&$ Sons 3 Nakayama, K. (1985) Vision Res. 25, 625-660

4 Poggio, T. and Reichardt, W. (1973) Kybernetik 13, 223-227

5 Buchner, E. (1984) Photoreception and Vision in Invertebrates (Ali, M. A., ed.), pp. 561-621, Plenum Press

6 Reichardt, W. (1987) J. Comp. Physiol. A 161, 533-547
7 Marr, D. and Ullman, S. (1981) Proc. R. Soc. London Ser. B. 211, 151-180

8 Hildreth, E. C. and Koch, C. (1987) Annu. Rev. Neurosci. 10, $477-533$

9 Torre, V. and Poggio, T. (1978) Proc. R. Soc. London Ser. B. 202, 409-416

10 Grzywacz, N. M. and Koch, C. (1987) Synapse 1, 417-434

11 Ullman, S. (1983) Trends Neurosci. 6, 177-179

12 van Santen, J. P. H. and Sperling, G. (1984) J. Opt. Soc. Am. A 1, 451-473

13 Adelson, E. H. and Bergen, J. R. (1985) J. Opt. Soc. Am. A 2, 284-299

14 van Doorn, A. J. and Koenderink J. J. (1982) Exp. Brain Res. $45,179-188$

15 van Doorn, A. J. and Koenderink, J. J. (1982) Exp. Brain Res. 45, 189-195

16 van Santen, J. P. H. and Sperling, G. (1985) J. Opt. Soc. Am. A 2, 300-321

17 Wilson, H. (1985) Biol. Cybern. 51, 213-222

18 Baker, C. L., Jr and Braddick, O. J. (1985) Perception 14, 181-192

19 Barlow, H. B. and Levick, W. R. (1965) J. Physiol. (London) $178,477-504$

20 Egelhaaf, M., Borst, A. and Reichardt, W. J. Opt. Soc. Am. A (in press)

21 Srinivasan, M. V. and Bernard, G. D. (1976) Biol. Cybern. 21. 227-236

22 Movshon, J. A., Thompson, I. D. and Tolhurst, D. J. (1978) J. Physiol. (London) 283, 79-99

23 Baker, C. L., Jr and Cynader, M. S. (1986) J. Neurophysiol. 55, 1136-1152

24 Mikami, A., Newsome, W. T. and Wurtz, R. H. (1986) J. Neurophysiol. 55, 1308-1327

25 Kirschfeld, K. (1972) Information Processing in the Visual System of Arthropods (Wehner, R., ed.), pp. 61-74, SpringerVerlag

26 Riehle, A. and Franceschini, N. (1984) Exp. Brain Res. 54, 390-394

27 Enroth-Cugell, C. and Robson, J. G. (1966) J. Physiol. (London) 187, 512-552

28 Shapley, R. M. and Hochstein, S. (1975) Nature 256, $411-413$

29 Movshon, J. A., Thompson, I. D. and Tolhurst, D. J. (1978) J. Physiol. (London) 283, 53-77

30 Reichardt, W. and Egelhaaf, M. (1988) Biol. Cybern. 58, 287-294

31 Zeki, S. (1983) Proc. R. Soc. London Ser. B. 217, 449-470

32 Felleman, D. J. and Kaas, J. H. (1984) J. Neurophysiol. 52, 488-513

33 Hausen, K. (1984) Photoreception and Vision in Invertebrates (Ali, M. A., ed.), pp. 523-559, Plenum Press

34 Lappin, J. S. and Bell, H. H. (1976) Vision Res. 16, 161-168 35 Chang, J. J. and Julesz, B. (1983) Vision Res, 23, 639-646

36 van Doorn, A. J. and Koenderink, J. J. (1984) Vision Res. 24, $47-53$

37 Hubel, D. H. and Wiesel, T. N. (1962) J. Physiol. (London) $160,106-154$

38 Holub, R. A. and Morton-Gibson, M. (1981) J. Neurophysiol $46,1244-1259$

39 Hausen, K. (1982) Biol. Cybern. 45, 143-156

40 Marmarelis, P. Z. and McCann, G. D. (1973) Kybernetik 12 74-89

41 Emerson, R. C., Citron, M. C., Vaughn, W. J. and Klein, S. A (1987) J. Neurophysiol. 58, 33-65

42 Shannon, C. E. and Weaver, W. (1949) The Mathematical Theory of Communication The University of Illinois Press

43 Varjú, D. (1959) Z. Naturforsch. 14b, 724-735

44 Götz, K. G. (1965) Kybernetik 2, 215-221

45 Burr, D. and Ross, J. (1986) Trends Neurosci. 9, 304-307

46 van de Grind, W. A., Koenderink, J. J. and van Doorn, A. J. (1986) Vision Res. 26, 797-810

47 Rodieck, R. W. (1973) The Vertebrate Retina W. H. Freeman

48 Chang, J. J. and Julesz, B. (1983) Vision Res. 23, 1379-1385

49 Nakayama, K. and Silverman, G. H. (1985) J. Opt. Soc. Am. A 2, 267-274

50 Pantle, A., Lehmkuhle, S. and Candill, M. (1978) Perception 7, 261-267

51 Anderson, S. J. and Burr, D. C. (1985) Vision Res. 25 , 1147-1154

52 Götz, K. G. (1964) Kybernetik 2, 77-92

53 Götz, K. G. (1972) Bibliotheca Ophthalmologica 82, 251-259
Acknowledgements

We thank Jürgen

Bolz, Karl G. Götz

Werner Reichardt

and Johannes $M$.

Zanker for critically reading the

manuscript, Shirley

Würtz for correcting

our English, and Frank

Buchstäber and Birgit

Pilz for drawing the

figures. 
54 Watanabe, A., Mori, T., Nagata, S. and Hiwatashi, K. (1968) Vision Res. 8, 1245-1263

55 Tolhurst, D. J., Sharpe, C. R. and Hart, G. (1973) Vision Res 13, 2545-2555

56 Pantle, A. (1974) Vision Res. 14, 1229-1236

57 Kelly, D. H. (1979) J. Opt. Soc. Am. 69, 1340-1349

58 Burr, D. C. and Ross, J. (1982) Vision Res. 22, 479-484

59 Wright, M. J. and Johnston, A. (1985) Vision Res. 25, 1947-1955

60 Miles, F. A. and Kawano, K. (1987) Trends Neurosci. 10, $153-158$

61 Egelhaaf, M. and Reichardt, W. (1987) Biol. Cybern. 56, 69-87

62 Egethaaf, M. and Borst, A. (1989) J. Opt. Soc. Am. A 6, 116-127

63 Borst, A. and Bahde, S. (1986) Biol. Cybern. 55, 59-69
64 Eckert, H. and Hamdorf, K. (1981) J. Comp. Physiol. A 145, 241-247

65 Moulden, B. and Begg, H. (1986) Perception 15, 139-155

66 Mather, G. (1984) Vision Res. 24, 1399-1405

67 Bischof, W. F. and Groner, M. (1985) Vision Res. 25 839-847

68 Koch, C., Poggio, T. and Torre, V. (1986) Trends Neurosci. 9. 204-211

69 Ariel, M. and Daw, N. W. (1982) J. Physiol. (London) 324 161-185

70 Werblin, F., Maguire, G., Lukasiewicz, P., Eliasof, S. and Wu S. M. (1988) Vis. Neurosci. 1, 317-329

71 Sillito, A. M. (1979) Trends Neurosci. 2, 196-198

72 Schmid, A. and Bülthoff, H. (1988) Biol. Cybern. 59, 71-80

73 Egelhaaf, M. and Borst, A. (1989) Dynamics and Plasticity in Neuronal Systems (Elsner, N. and Singer, W., eds), p. 106, Georg Thieme Verlag

\section{books}

\section{Cell Culture Approaches to Invertebrate Neuroscience}

\author{
Chris Reddies \\ Ron McKay \\ Department of Brain \\ and Cognitive \\ Science, \\ Massachusetts \\ Institute of \\ Technology, \\ Room E25-435, \\ 45 Carleton Street, \\ Cambridge, $M A$ \\ 02139, USA.
}

edited by D. J. Beadle, G. Lees and S. B. Kater, Academic Press, 1988. $f 24.50$ (240 pages) ISBN 012 $083510 x$

Scientists studying the invertebrate nervous system are quick to point out how much less complex the nervous system of invertebrates is compared with the brain of higher organisms. In this book, the 'simple' invertebrate nervous system is taken to an even more basic level, cell culture.

In eight chapters, experts in the field review their work on cultured neurons from pond snails, leeches, sea slugs, cockroaches, fruit flies and grasshoppers. The chapters on insects deal mostly with issues of membrane excitability and neurotransmitter systems, whereas the others focus on developmental issues like neurite outgrowth and synaptogenesis. All the chapters provide ample evidence of the two main advantages of cell culture. First, cells growing on the surface of a dish are easily accessible and allow studies which would be difficult or impossible to perform in the living animal. Second, the environment of the culture dish is well defined and can be manipulated to study neuronal behavior. Thus, the complexity of the nervous system in vivo can be reduced to welldefined components in the culture dish.

The methods described in this book are as diverse as the species used. They range from time-lapse video-microscopy of intracellular calcium concentrations in living growth cones, to the mutational analysis of membrane excitability in Drosophila neural cultures, to single-channel recordings from myosac membranes. Results obtained in cell culture are compared with in vivo findings to which they often correspond in surprising detail.

This book meets its goal of giving an up-to-date review of invertebrate neuron culture. It is highly recommended to the novice who wants to get an introduction into the field. The expert may find it useful as a reference that gathers much information in one place. The use of the book as a reference in the laboratory is limited since some chapters do not give enough detail or are poorly illustrated in the methodology sections. Researchers who do not work with invertebrate neurons may want to read individual chapters in this book because they show how elegantly so many different aspects of neuroscience can be tackled in a system as basic as invertebrate neuron culture. Most chapters are concise and easily readable. However, the general relevance of the material presented in this multi-authored book is not the same in each chapter. While most of the chapters do stress concepts and provide examples of how this new methodology can lead to new insight into the nervous system, there are also one or two chapters that describe results without really providing much perspective on conceptual advances. The many figures in the book enhance its value. Excellent black-and-white photomicrographs show the complex patterns of neurite formation in the culture dish but seem pale compared with their full beauty when viewed under the microscope in the laboratory.

The case for the usefulness of the cell culture approach is made in several chapters of the book. Particularly convincing are the studies on the growth cones of identified neurons of the pond snail Heliosoma. The ease of cell culture differs for the various preparations described. Molluscan neurons grow readily in culture. Kater and his colleagues have pioneered the characterization of this system. One of their most striking findings was that the growth cones of identified neurons had different morphologies and responses to neurotransmitters. The different behaviors of growth cones appear to be regulated by intracellular calcium levels. The other preparations described focus on different questions. In Aplysia, the focus is on synapse formation and modulation. The leech studies are directed at the question of neuronal wiring, and the Drosophila studies are focused on the cellular mechanism linking genetic change to a whole animal phenotype. The authors' enthusiasm for their projects is infectious.

So far, cell culture methods have focused on elementary topics like neurite outgrowth, specificity of synapse formation, and membrane excitability. But can invertebrate cell culture bring together the individual components and help in the study of the behavior of complex circuits? After reading this book, one wonders whether the invertebrate nervous system can really be constituted in a dish? 